Journal of Advanced College of Engineering and Management, Vol.1, 2015

\title{
FUZZY CLUSTERING BASED BLIND ADAPTIVE OFDM SYSTEM
}

\author{
Krishna Sundar Twayana ${ }^{1}$, Shashidhar Ram Joshi ${ }^{2}$ \\ ${ }^{1}$ Department of Electronics and Computer Engineering, Advanced College of Engineering \& \\ Management, Kopundole, Nepal \\ Email Address: krishna.twayana@acem.edu.np \\ ${ }^{2}$ Department of Electronics and Computer Engineering Institute of Engineering, Pulchowk Campus \\ Email Address: sashi@healthnet.org.np
}

\begin{abstract}
This paper present blind adaptive modulation for OFDM system. Automatic recognition of the modulation level of detected signal and blind estimation of SNR is a major task of an intelligent receiver presented here. In this paper, modulation classification is performed by fuzzy c-means clustering followed by new approach of modulation classification, fuzzy template matching considering the constellation of the received signal. In addition, SNR estimation and modulation order for the next transmission is carry out by Fuzzy Logic Interface based upon heuristics. The simulation that has been conducted shows high capability of recognition of modulation level, almost perfect estimation of SNR and adaptive with considerable BER in an Additive White Gaussian Noise channel. The performance of proposed system is simulated in computer simulation tool, MATLAB 7.0.
\end{abstract}

Keywords: Adaptive modulation, SNR estimation, Fuzzy c-means clustering, Fuzzy template matching.

\section{Introduction}

Adaptive transmission is real-time balancing of the link budget through adaptive variation of the transmitted power level, symbol transmission rate, constellation size, coding scheme, or combination of these parameters without sacrificing the BER. These schemes provide a higher average link spectral efficiency, which require channel estimation at the receiver.

In modern wireless digital communication, the precise knowledge of SNR is important. Many algorithms require the knowledge of SNR for their optimal performance. In data-aided (DA) SNR estimation also known as pilot symbol assisted modulation (PSAM), SNR of the signal through rapid fading environment is detected by periodically inserting known symbols [1], from which the receiver derives its amplitude and phase reference. However, the training sequence limits system throughput rate. Where as in non-data aided (NDA) SNR estimation system [2, 3, instantaneous SNR is compute from the received signal and the receiver feedback appropriate transmission parameter to adapt into future channel conditions.

Clustering algorithms have been studied for a long time, and are used nowadays for a large number of applications. They are successfully in use in systems for information access, data mining or computer vision. Different algorithms have been developed using different approaches 
and considering different underlying assumptions on the data and on the final set of clusters. Fuzzy clustering [4, 5] is one of the well known robust and efficient approach to reduce computation cost to obtain the better results. Fuzzy clustering can obtain not only the belonging status of objects but also how much the objects belong to the clusters. The boundaries between clusters could not be defined precisely, some of the data could belong to more than one cluster with different positive degrees of memberships. This clustering method considers each cluster as a fuzzy set and the membership function measures the degree of belonging of each feature in a cluster. So, each feature may be assigned to multiple clusters with some degree of belonging. The aim of modulation classification (MC) [4, 5, 6, 7] is to identify the modulation type of a communication signal. This is a challenging problem, especially in non-cooperative environments, where no prior knowledge on the incoming signal is available.

\section{Methodology}

\subsection{Complete System}

The proposed system as shown in Fig 1, is based on a statistical ratio of observables over a block of data. Average deviation, which has direct impact on bit error rate, determine the appropriate modulation order for the next transmission frame. Rules are defined in such a way that modulation order switch to new one based on bit error rate for particular modulation order at given SNR. The receiver feedback appropriate modulation level to the transmitter for subsequent data transmission through the channel.

The proposed technique has been designed so that it would be capable of recognizing the types: 4-QAM, 8-QAM, 16-QAM, 32-QAM, 64-QAM, 128-QAM and 256-QAM. In order to reduce complexity, all the received symbols have been mapped and 64 cluster center have been randomly initialized in the first quadrant. Fuzzy c-means clustering compute the centroids of a cluster as being the mean of all points weighted by their degree of belonging to the cluster iteratively until program reach some termination condition. Upon completion of the fuzzy algorithm centroids are obtained and then fuzzy template matching process is done for modulation classification. The automatic recognition of the modulation format of a detected signal is the intermediate step between signal detection and demodulation, which is a major task of an intelligent receiver.

\subsection{Fuzzy Logic SNR and Modulation Estimation}

The non data aided fuzzy logic SNR estimator and modulation order controller for QAM signals is derived base on a statistical ratio of observables over a block of data. The average deviation of real and imaginary components from its likelihood values and present modulation order obtained after modulation classification, Fuzzy Inference System (FIS) estimates the SNR value and best modulation order for next transmission frame to maintain BER below $10^{-3}$ from rules and membership functions defined for average deviation and present modulation order.

\subsection{Fuzzy C-Means Clustering}

Fuzzy c-means (FCM) is a method of clustering which allows one piece of data to belong to two or more clusters. This method is frequently used in pattern recognition. It is based on 


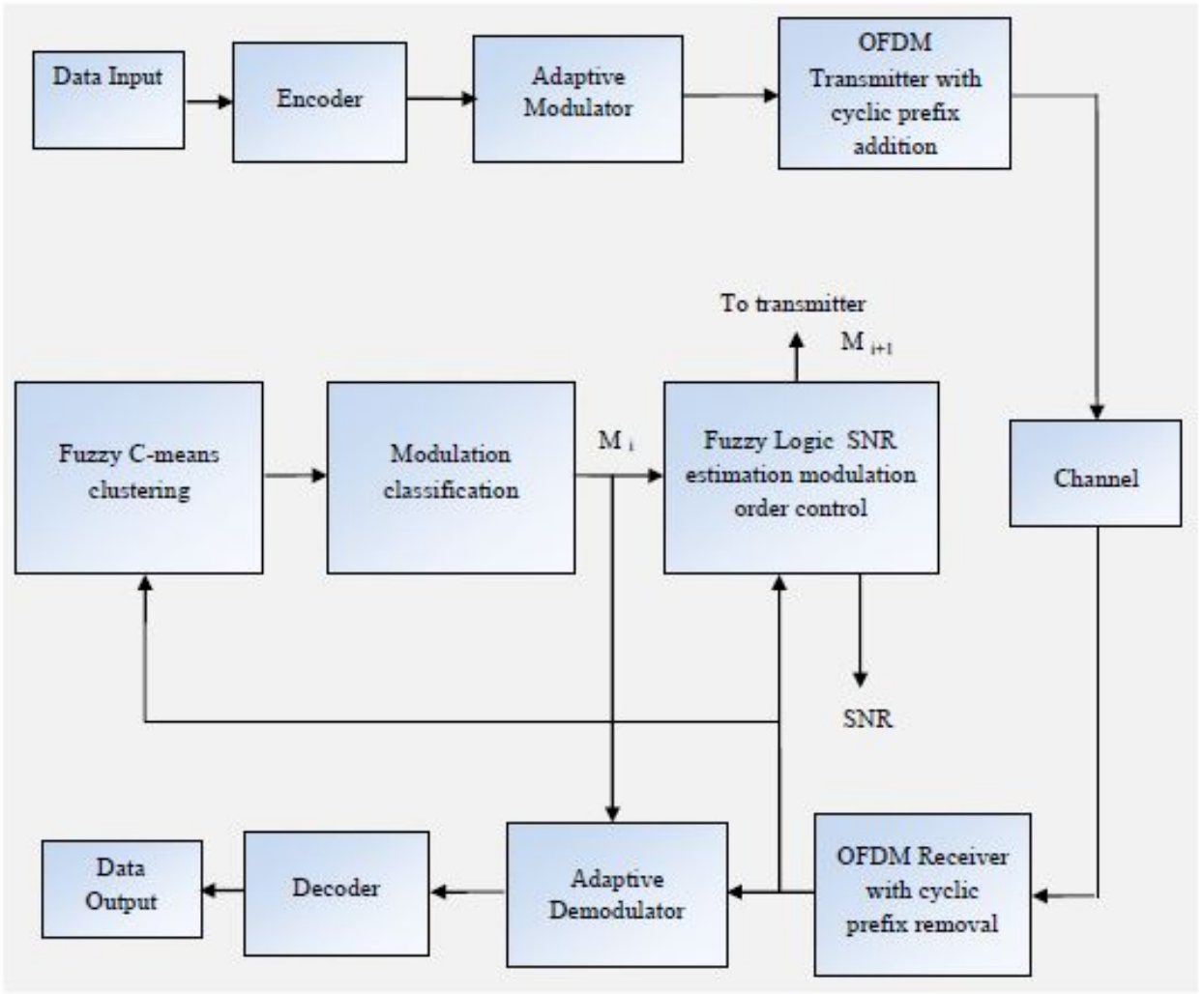

Fig 1: Complete Diagram of Proposed System

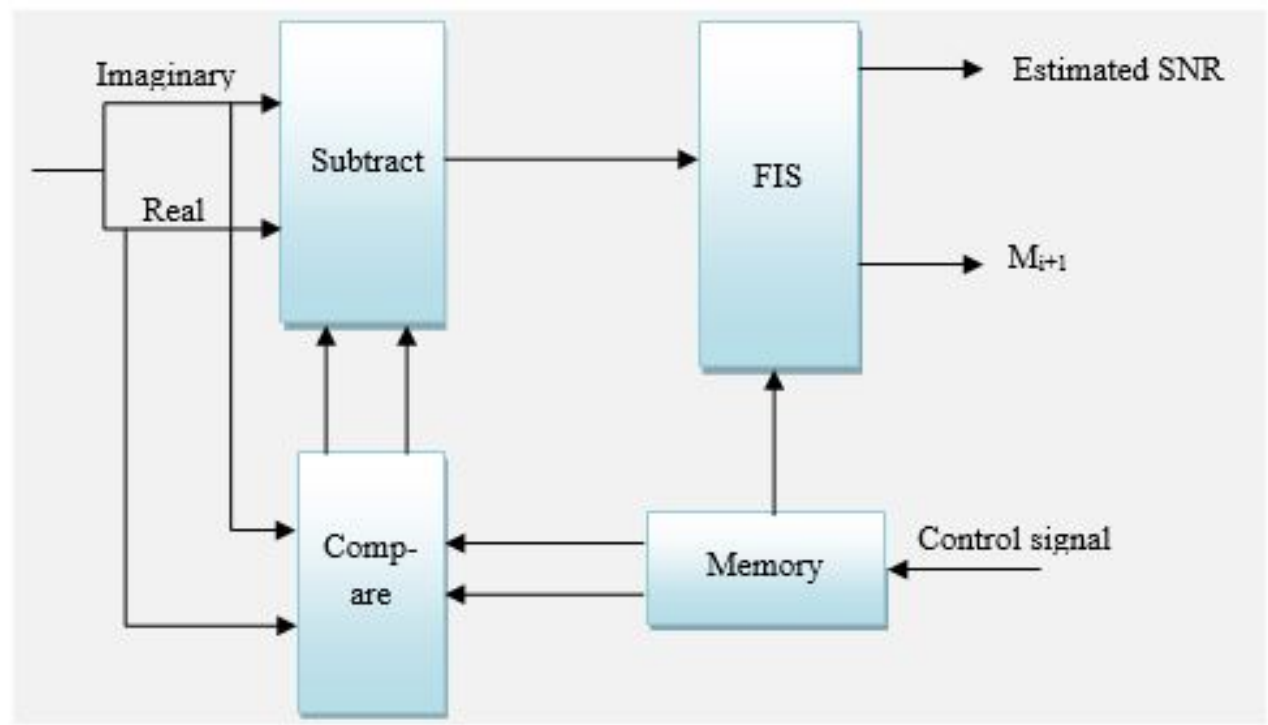

Fig 2: Fuzzy Logic SNR Estimation and Modulation Control 
minimization of the following objective function:

$$
\jmath=\sum_{k=1}^{N} \sum_{i=1}^{C} \mu_{i k}^{m}\left\|x_{k}-c_{i}^{2}\right\|, \quad 1 \leqslant m<\infty
$$

where $m$ is any real number greater than $1, \mu_{i k}$ is the degree of membership of $x_{k}$ in the cluster $c_{i}, x_{k}$ is the $i^{t h}$ of D-dimensional measured data, $c_{i}$ is the D-dimension center of the cluster, and $\|*\|$ is any norm expressing the similarity between any measured data and the center. Fuzzy partitioning is carried out through an iterative optimization of the equation 1, with the update of membership $\mu_{i k}$ and the cluster centers $c_{i}$ as steps shown below:

i. Initialize $U=\left[\mu_{i k}\right]$ matrix, $U^{(0)}$

ii. At j step: calculate the centers vectors $C^{(j)}=\left[c_{i}\right]$ with $U^{(j)}$

$$
c_{i}=\frac{\sum_{k=1}^{N} \mu_{i k}^{m} * x_{k}}{\sum_{k=1}^{N} \mu_{i k}^{m}}
$$

iii. Update $U^{(j)}, U^{(j+1)}$ :

$$
\mu_{i k}=\frac{1}{\sum_{j=1}^{c}\left(\frac{\left\|x_{k}-c_{i}\right\|}{\left\|x_{k}-c_{j}\right\|}\right)^{\frac{2}{m-1}}}
$$

iv. Calculate $j^{(j)}$ from equation 1

v. If $\left\|\jmath^{(j)}-\jmath^{(j-1)}\right\|<\varepsilon$ then STOP; otherwise return to step ii.

\subsection{Fuzzy Template Matching}

The main idea in this method is assessment of resulted cluster centers from fuzzy c-means clustering based on relative similarity that exist between different kind of standard QAM modulations with predefined levels. In the beginning for every kind of QAM modulation family, the ideal centriods in first quadrant have been defined. It must be mentioned that, since the value of the objective function decrease naturally as the number of clusters increases, which in turn causes an error in recognition of the actual number of cluster, the value of the objective function is multiplied by a weight proportional to the number of clusters to prevent producing error. After multiplication of the weights, the number of clusters corresponding to the cost function with minimum value is chosen as the final number of cluster. After the final clusters on the first quadrant are obtained, these clusters are extended to the whole constellation diagram.

Fuzzy Template Matching algorithm for modulation classification:

i. Initialize ideal cluster centers for M-QAM $C_{j}, \quad 1 \leqslant j \leqslant \frac{M}{4}$

ii. Calculate the Euclidean distance of the clusters pair-wise between $C_{j}$ and cluster centers from fuzzy c-means clustering $c_{i}, 1 \leqslant i \leqslant 64$

$$
d_{i j}=\left\|c_{i}-C_{j}\right\|
$$


iii. Compute membership values

$$
\mu_{j i}=\frac{1}{\sum_{k=1}^{C}\left(\frac{\left\|c_{i}-C_{j}\right\|}{\left\|c_{i}-C_{k}\right\|}\right)^{\frac{2}{m-1}}}
$$

iv. Evaluate cost function:

$$
\text { cost_fun }_{-} \min (d(i,:)) * \max (\mu(:, i)) * \frac{M}{4}
$$

v. Repeat steps i to iv for all considered modulation order

vi. Select the modulation order corresponding to the minimum cost function

\section{Result and Discussion}

\subsection{Overall Simulation Result}

In order to evaluate the performance of the proposed system, simulation has been performed for various SNR values and different levels of QAM modulations on randomly generated binary data stream. Channel model apply for this system is assumed quasi-stationary AWGN channel, and it is assumed that there is no time and/or frequency synchronization error also. Fuzzy c-means clustering algorithm has been used for constellation recovery and modulation classification is performed to achieve the actual number of cluster centers. SNR estimation and change of modulation order are carried out by FIS. Simulation result in command window is shown in Fig 3 .

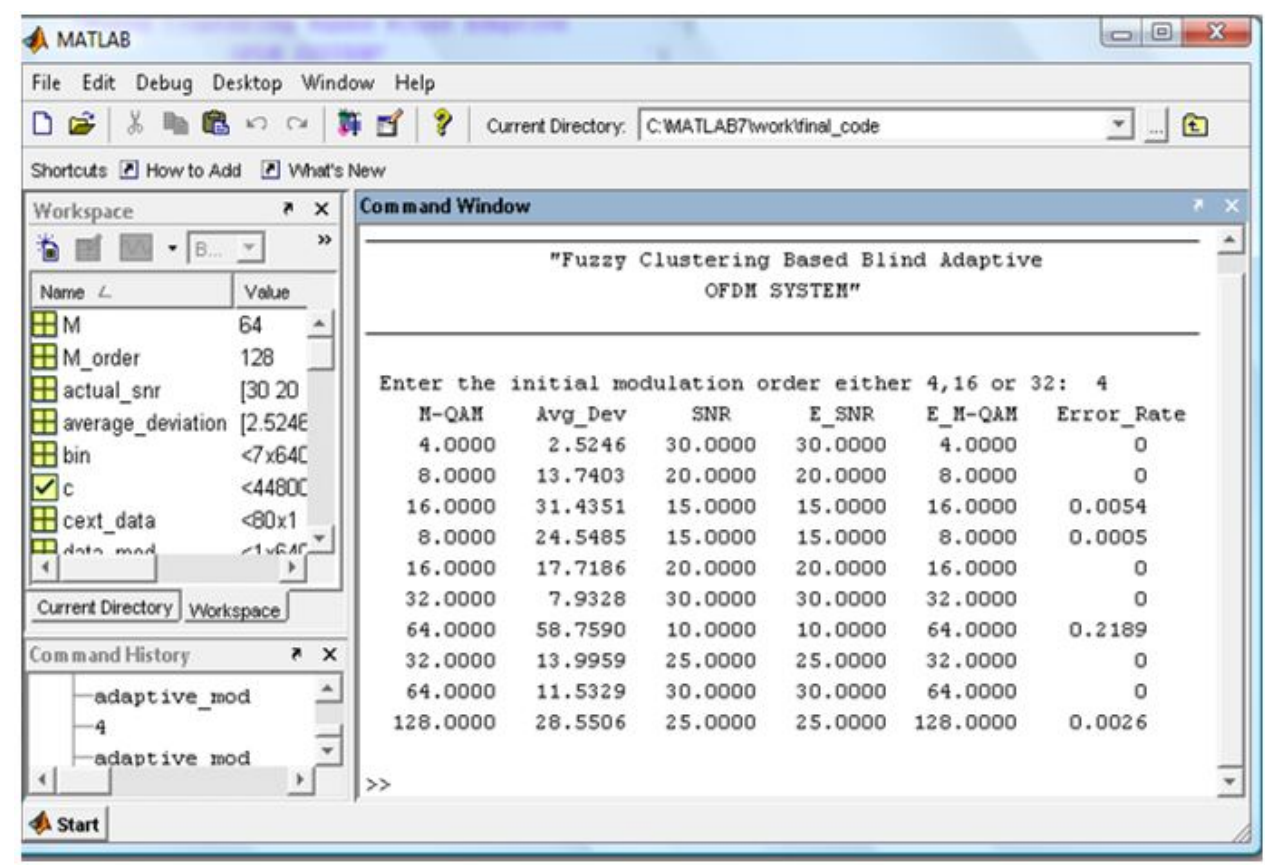

Fig 3: Overall Simulation Result 


\subsection{SNR Estimation}

Non data aided SNR estimation implemented in this paper is based on average deviation of in-phase and quadrature component of received signal at given modulation level. Using fuzzy logic in decision making is a good choice because ordinary (non fuzzy) system is controlled by plain if and else statements, for example if estimated SNR $=10 \mathrm{~dB}$ for average deviation in $(26$, 24) range is declared in the algorithm and if we get average deviation 26.1, using plain rule based system will yields an error whereas using fuzzy logic system will yield output SNR near to 10dB. So the estimated SNR by fuzzy logic is more likely to the actual SNR value in contrast to that by non fuzzy system as shown in Fig 4.

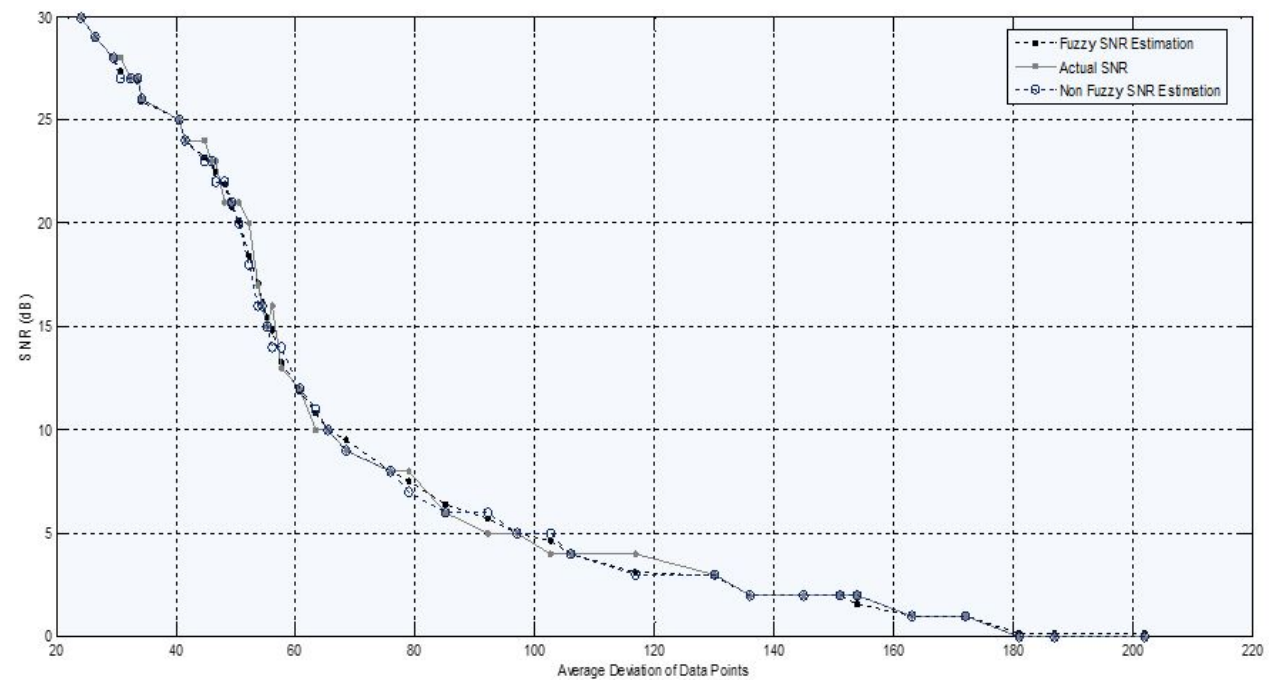

Fig 4: Comparison of Estimated SNR and Actual SNR

\subsection{Modulation Classification}

After the centroids are obtained from fuzzy c-means clustering, modulation type is recognized by evaluating the amount of matching with the standard templates of QAM modulation. Simulation result shows the proposed method have good recognition performance even in low SNR condition. The performance of the FCM algorithm depends on the selection of the initial cluster center and/or the initial membership value and fuzzifier $m$ determines the level of cluster fuzziness. In this paper the initial number of clusters has been set to 64 in the first quadrant randomly and fuzzifier $m$ is set to 2 for clustering. Fig 5 below shows simulation results of recognition of 16-QAM modulation levels with $5 \mathrm{~dB}$ SNR value by taking 500 samples where captions are:

a. Centroids in the first quadrant obtained from fuzzy clustering

b. Values of the objective functions for possible clusters in first quadrant

c. Centroids of the clusters in whole constellation diagram

d. Extended clusters and their centroids in whole constellation diagram

The accuracy of recognition versus SNR ratio for different modulation types of QAM family is shown in Fig 6. This method can recognize all the 4-QAM modulations with $100 \%$ accuracy 


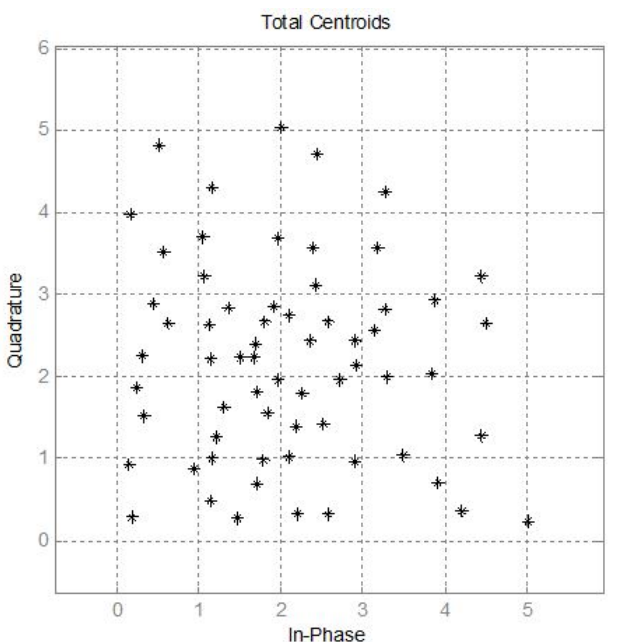

(a)

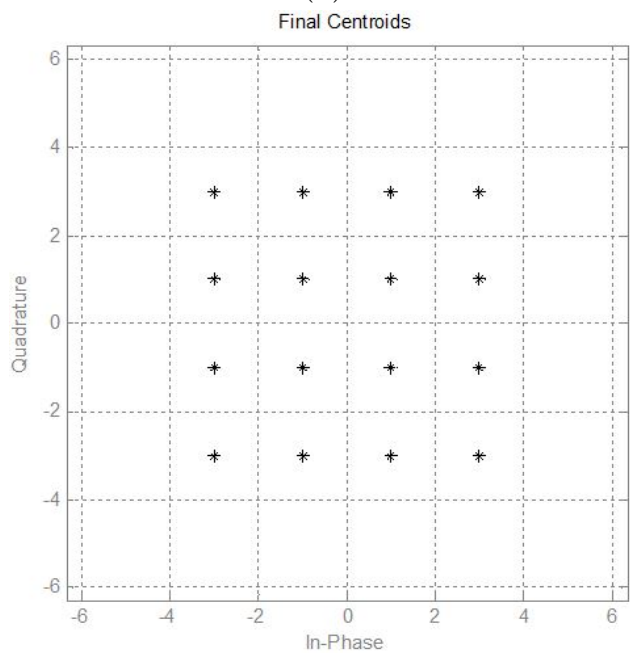

(c)

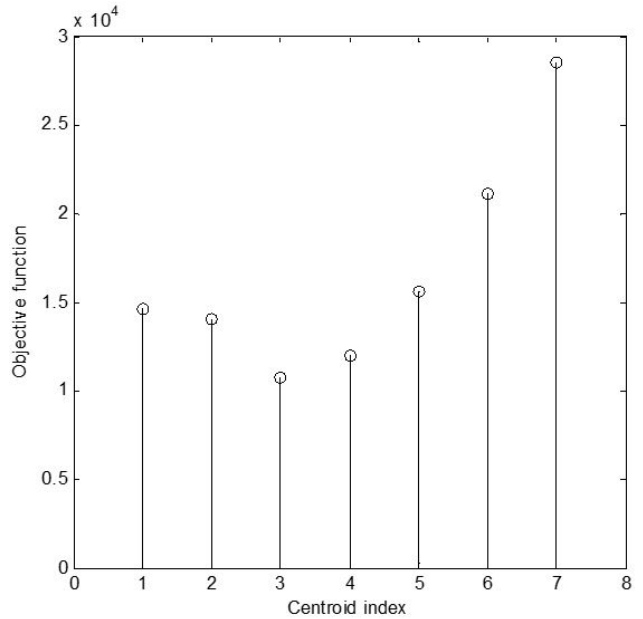

(b)

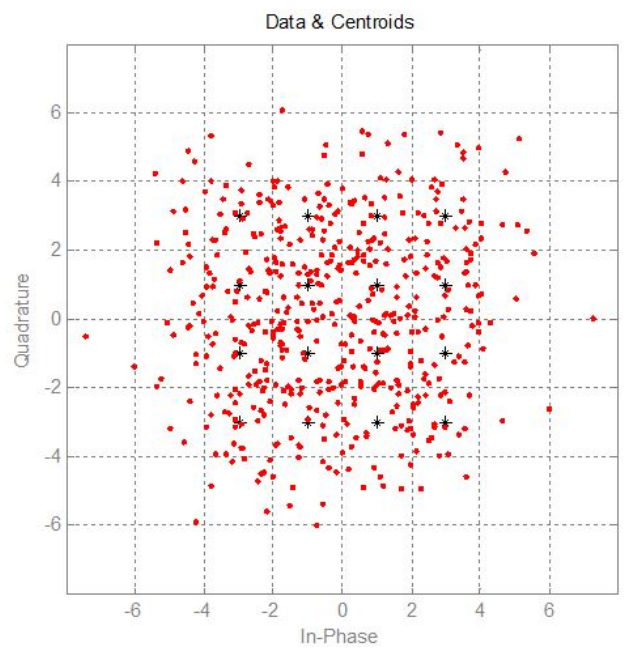

(d)

Fig 5: Recognition of 16-QAM with $\mathrm{SNR}=5 \mathrm{~dB}$

and its recognition truth does not depend on the signal to the noise, and it can also identify this modulation type with $\mathrm{SNR}=0 \mathrm{~dB}$. Similarly, this method can identify all the 16-QAM modulation with $\mathrm{SNR}=7 \mathrm{~dB}$ with $100 \%$ accuracy. Nevertheless, accuracy of recognition of 64 QAM and 128-QAM for the SNR 10dB and higher than that is $100 \%$ and for a level lower than it is less. Accuracy of recognition of 256 QAM is better than other modulation levels except of 4 QAM.

\section{Conclusion}

The results of computer simulation shows that almost perfect SNR estimation and modulation recognition even in low SNR condition and system is adaptive with noise condition. In the new fuzzy template matching algorithm, there is no restriction on order of assessment to be started from highest level of 256 QAM and end at lowest level 4 QAM as in normal template matching. This approach could be extended and modified to recognize other types of digital modulations in different fading channels. 


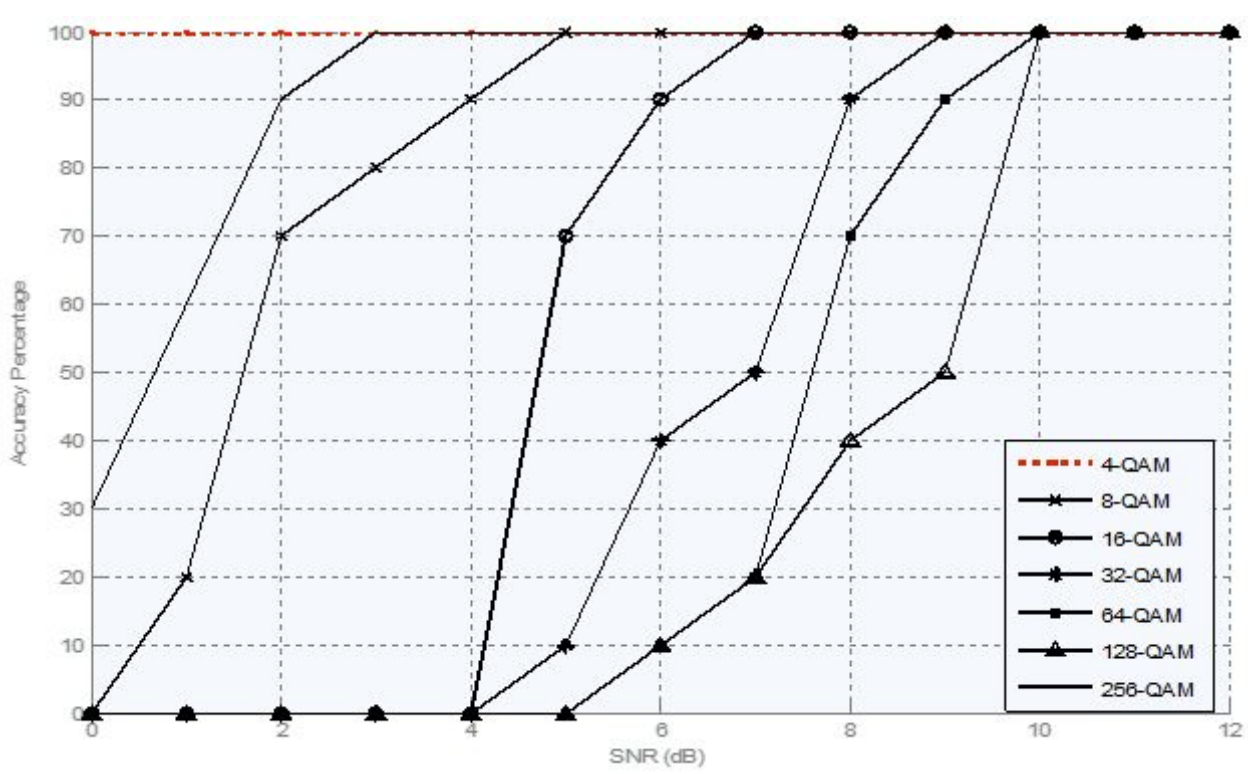

Fig 6: Accuracy of QAM Modulation Recognition Versus SNR

\section{References}

[1] K.Seshadri Sastry, Dr. M.S.Prasad Babu, "Adaptive Modulation for OFDM system using Fuzzy logic interface." IEEE Conference proceedings, IEEE International Conference in Software Engineering and Service Sciences, Beijing, China.

[2] K.Seshadri Sastry, Dr. M.S.Prasad Babu, "SNR Estimation For QAM signals Using Fuzzy Logic Interface." IEEE Conference proceedings, IEEE International Conference in Computer Science and Information technology, Chengdu , China.

[3] K.Seshadri Sastry, Dr. M.S.Prasad Babu, "Fuzzy logic based Adaptive Modulation Using Non Data Aided SNR Estimation for OFDM system," International Journal of Engineering Science and Technology, vol. 2(6), pp. 2384-2392, 2010.

[4] Bijan G Mobaseri, "Constellation shape as a robust signature for digital modulation recognition," Military Communications Conference Proceedings, MILCOM IEEE, 1:442-446, 1999.

[5] Bijan G Mobaseri, "Digital Modulation Classification using Constellation Shape," signal processing, 1:442-446, 1999.

[6] Negar Ahmadi, Reza Berangi, "A Template Matching Approach to Classification of QAM Modulation using Genetic Algorithm," Signal Processing: An International Journal, vol. 3 Issue 5, pp. 95-109, 2009.

[7] Negar Ahmadi, Reza Berangi, "Symbol Based Modulation Classification using Combination of Fuzzy Clustering and Hierarchical Clustering," Signal Processing: An International Journal, vol. 4,Issue 5, pp. 95-109, 2010. 